\title{
Study on the strategy of three-dimensional anatomy of the duodenum and its surrounding organs
}

\author{
Chengan Feng ${ }^{1, \mathrm{a}}$, Yan Fan ${ }^{2, \mathrm{~b}}$ (Corresponding author) \\ ${ }^{1}$ Department of Anatomy and Histology \& Embryology, School of Basic Medical Science, Kunming Medical University \\ ${ }^{2}$ Department of Anatomy and Histology \& Embryology, School of Basic Medical Science, Kunming Medical University
}

\begin{abstract}
With the development of science and technology, there are constantly new technologies used in medicine, and related medical technologies have also been greatly improved. Anatomy is one of them. This technology has been widely used in clinical practice, but traditional anatomy is evaluated before surgery. There are shortcomings, and it is impossible to have a more accurate understanding of the disease. Therefore, improper operation may still cause some problems during the operation, such as bile duct and blood vessel damage, organ failure and other adverse consequences. Duodenum and its surrounding organs are a complex surgical method. Based on the characteristics of the site, it involves multiple organs such as the pancreas, duodenum, and bile ducts. The anatomical structure of the organism is destroyed, and the destroyed structure is artificially constructed to create a highly difficult surgical technique for new physiological channels. With the development of medicine, the application of three-dimensional visualization technology in this operation can accurately measure the pathology of the duodenum and its surrounding organs, and make preoperative planning and planning for the excision of the duodenum and its surrounding organs. The success of the operation plays an important role. This article mainly analyzes the current situation of the anatomy of the duodenum and its surrounding organs under the background of current medical technology, and proposes an idea on how to optimize and enhance the anatomy of the duodenum and its surrounding organs. Series recommendations. And try to find a new way out for the anatomy of the duodenum and its surrounding organs in the current era based on the results of these studies. Experimental research shows that the use of three-dimensional technology can greatly improve the anatomy of the duodenum and its surrounding organs. The use of this technology, combined with the doctor's solid foundation, can greatly improve the success rate of the operation.
\end{abstract}

\section{Introduction}

Resection surgery is currently recognized worldwide as the most effective treatment for many malignant diseases. Through the regular resection of the diseased organ, the diseased organ can be completely removed without affecting the basic body functions of the person. However, with the irregular resection, more functional tissues are lost. Patients with weak physical fitness especially face a greater risk of postoperative organ failure [1]. In medical treatment, through traditional CT, MRI and other imaging technologies, doctors combined with rich clinical experience can also predict the shape and size of the lesion to a certain extent, but because of the limitations of this technology, there are still Variations of some blood vessels and bile ducts which cannot be detected in advance, and there will still be many emergencies during the operation [2]. Some doctors do not have enough experience to fully grasp the spatial relationship between the pathogen and the surrounding blood vessels and related structures. Therefore, the formulation of the surgical plan will also be unreasonable. The blindness and risk of the operation also exist, and there are some deviations in the precise resection. In the course of the operation, other injuries are caused to the patient, and complications may occur in serious cases [3].

Three-dimensional visualization technology can greatly improve anatomical resection from a technical level [4]. The volume of the duodenum and its surrounding organs and the ratio of the volume of the duodenum and its surrounding organs can be calculated using this technology before surgery. It can be analyzed from many aspects of organ reserve function. Predict the surgical risk in advance, reduce the surgical risk, evaluate the probability of postoperative risk, and effectively improve the safety of surgery [5]. At the same time, when performing preoperative predictive analysis, patients and their families can intuitively understand the surgical plan and surgical risks, which is beneficial to promote communication between doctors and patients and reduce unnecessary medical disputes [6].

In the current era, medical technology still has many shortcomings. For example, the application technology of imaging technology in medicine is not yet mature, and the pipeline structure in the organ is too small, so

afengca@126.com, ${ }^{b}$ fengca@126.com 
problems can only be discovered and solved in time during surgery. In clinical practice, it is often difficult for doctors to obtain ideal images of bile ducts in organs [7-8]. Although 3D visualization technology is a relatively mature technology at present, it still has limitations and shortcomings. Doctors should not blindly rely on this technology in clinical practice, and they need to have certain knowledge and rich clinical experience. Blindly relying on three-dimensional visualization technology and ignoring the doctor himself, this will cause judgment and surgical deviation to a certain extent, which is not conducive to the operation [9]. Only on the basis of obtaining reliable and high-quality CT image data, combining the doctor's solid foundation and rich clinical experience, accurately identifying the problematic organ during the operation, and performing resection, can it be possible to maximize the use of 3D visualization technology clinical value advantage [10].

\section{Method}

\subsection{Preoperative analysis}

Preoperative comprehensive analysis and discussion of the patient's medical history, combined with three-dimensional visualization technology to accurately assess the patient's current condition, clarify the function of the duodenum and its surrounding organs, and analyze the duodenum and its surrounding organs before the operation anatomy and variation. There is also an accurate risk assessment of this operation, combined with the three-dimensional visualization model for doctors to study and discuss, and work out the best surgical plan.

\subsection{Statistical methods}

The SPASS19.0 statistical system was used for statistical analysis, the measurement data was using t-test, and the comparison of rates was using the PearsonX2 test. When the expected count is small (theoretical frequency $\mathrm{T}<5$ ), the Fisher's exact test is used. $\mathrm{P}<0.05$ indicates a significant difference. Statistical significance. If a function $g(f)$ satisfies the following relationship:

$$
\frac{\mathrm{P}_{\mathrm{r}}\left(\mathrm{x}_{\mathrm{i}}=0 \mid\{\mathrm{r}\}, \mathrm{S}\right)}{\mathrm{P}_{\mathrm{r}}\left(\mathrm{x}_{\mathrm{i}}=1 \mid\{\mathrm{r}\}, \mathrm{S}\right)} \geq 1
$$

Then the space formed by the function $g(f)$ is called the square integrable space, that is, $g(t) \in(R)$. From this formula, we can see that $g(t)$ is an energy finite function. If the function $y(f) \in(t)$, its corresponding Fourier transform is, when the condition is met:

$$
\frac{1}{2}\left(1+\left(1-2 \mathrm{P}_{\mathrm{j}}^{\mathrm{i}}\right)\right)
$$

It is called a basic wavelet function. After the function is translated and stretched, the result is a wavelet sequence:

$$
\mathrm{R}_{\mathrm{i}-\mathrm{j}}^{0}=\frac{1}{2}\left(1+\left(1-2 \mathrm{P}_{\mathrm{j}}^{\mathrm{i}}\right)\right)
$$

The above probability is:

$$
\mathrm{R}_{\mathrm{i}-\mathrm{j}}^{1}=1-\mathrm{R}_{\mathrm{i}-\mathrm{j}}^{0}
$$

The above formula leads to the following ruling:

$$
\left\{\begin{array}{l}
x_{j}=0 \\
x_{j}=1
\end{array}\right.
$$

\subsection{Tree-D model establishment}

Since the beginning of the 21st century, 3D printing (3DP) technology has been applied in the medical field. 3D printing technology is the opposite of traditional material removal processing methods. It is based on a three-dimensional digital model and usually uses a layer-by-layer manufacturing method to combine materials. In recent years, 3DP technology has been widely used, such as personalized surgical planning and guiding medical research. Compared with $\mathrm{CT}$, magnetic resonance imaging (MRI) and other two-dimensional imaging, three-dimensional visualization (3Dv) and 3DP models are more intuitive and accurate in displaying the anatomical and spatial structures of organs, and have made great achievements. Three-dimensional visualization reconstruction is to use software to reconstruct a two-dimensional CT image into a three-dimensional visualization model. In order to better show the relationship between tumors or lesions and blood vessels and adjacent organs, 3D printing models can print complete models of tumors, surrounding organs, and large blood vessels, or only print models of selected organs and large blood vessels. Through the physical model and being able to touch the model, it is helpful for clinicians to closely observe the anatomical relationship of the tumor and make preoperative plans. They have been widely used in liver surgery, plastic surgery and orthopedics. However, the application of 3Dv and 3DP in the diagnosis of complex intestinal obstruction is still at an early stage, with few reports. We report a case of intestinal obstruction caused by internal hernia confirmed by $3 \mathrm{D}$ visualization technology. Due to timely diagnosis and timely surgery, the patient has a good prognosis.

\section{Experiment}

\subsection{Experimental investigation objects}

In order to be able to analyze the research of the three-dimensional anatomy program in more depth, this article has conducted investigations from both the patients and the doctors, through the anatomy treatment of the duodenum and surrounding organs of 40 patients in a hospital in the past month Understand the situation; and what doctors think about the use of 3D technology in surgery and the impact on surgery. Conduct research and investigation to understand the research results of the research subjects on the subject of this article. Based on the 3D technology Problems and causes in clinical medicine. Through comprehensive processing and analysis of data. 


\subsection{Experimental design}

After 40 patients were admitted to the hospital, a comprehensive examination was performed. All patients had no serious surgical contraindications, related matters that could be performed normally, and no related diseases that could not tolerate surgery. 40 patients were treated in groups. Divide them into experimental and control groups, each with 20 people. The experimental group used three-dimensional technology for surgery, while the control group did not use three-dimensional technology for surgery. When selecting 40 patients for surgery, statistics were made on the patients' operating time, surgical blood loss, and postoperative recovery. The experimental group established a three-dimensional reconstruction model for preoperative planning and intraoperative application, while the control group did not establish a three-dimensional reconstruction model for preoperative planning and intraoperative application.

\section{Results}

\subsection{Experimental results}

Table 1. Observation indicators of the two groups

\begin{tabular}{|c|c|c|c|}
\hline & test group & $\begin{array}{c}\text { Control } \\
\text { group }\end{array}$ & P value \\
\hline $\begin{array}{c}\text { Operation time } \\
\text { (min) }\end{array}$ & 164 & 205 & 0.016 \\
\hline $\begin{array}{c}\text { Surgery blood } \\
\text { loss (ml) }\end{array}$ & 240 & 420 & 0.011 \\
\hline
\end{tabular}

\begin{tabular}{|c|c|c|c|}
\hline $\begin{array}{c}\text { Postoperative } \\
\text { response time } \\
\text { (day) }\end{array}$ & 10 & 15 & 0.043 \\
\hline
\end{tabular}

Among the relevant indicators for the investigation of the anatomy of the duodenum and its surrounding organs, the average time spent in the experimental group for an anatomical operation of the duodenum and its surrounding organs was about 164 minutes, and the control The average time of the group was significantly reduced compared to $205 \mathrm{~min}$, so the difference was statistically significant $(\mathrm{P}=0.016)$. The average intraoperative blood loss during the operation of the experimental group was about $240 \mathrm{~mL}$, which was significantly reduced compared with the $420 \mathrm{~mL}$ of the control group. The difference was statistically significant $(\mathrm{P}=0.011)$. In terms of postoperative recovery, the average time for the experimental group to return to a normal state was 10 days; compared with the 15 days of the control group, it was also significantly reduced, and the difference was statistically significant $(\mathrm{P}=0.043)$. From the comparison between the experimental group and the control group, we can see that the experimental group has established a three-dimensional reconstruction model for preoperative planning and intraoperative application. The operation time, surgical blood loss and postoperative recovery are better than the experimental group, so it can It is concluded that three-dimensional technology has a huge improvement in the success rate of surgery, and can be used on a large scale in clinical surgery to reduce the problems that occur during surgery.

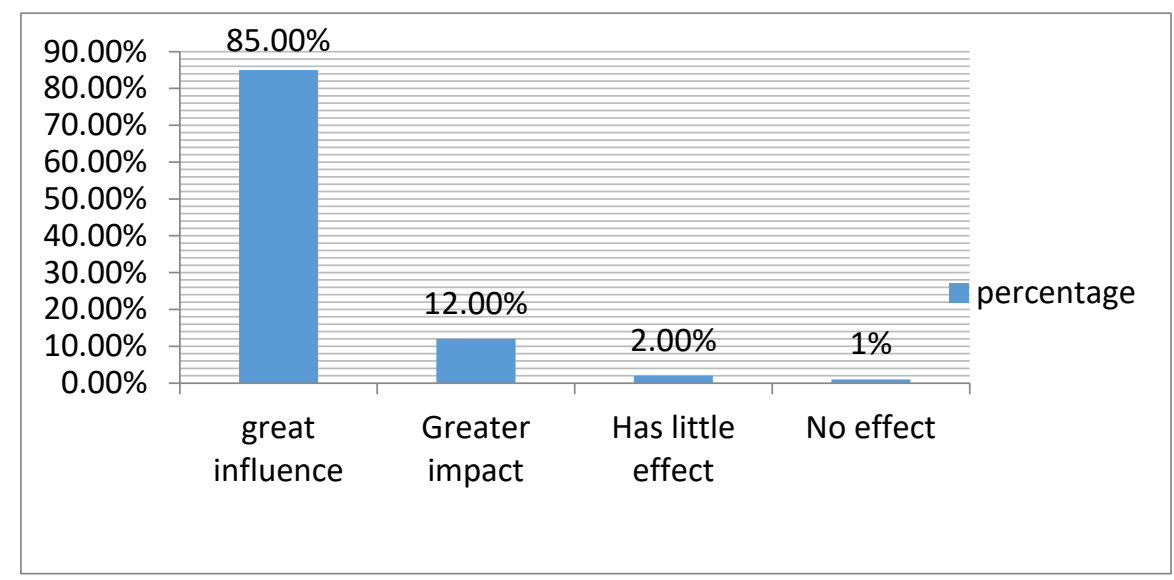

Figure 1. Does 3D technology affect the anatomy of the duodenum and its surrounding organs?

The data shown in Figure 1 is a survey of doctors in the hospital. From the data in the figure, it can be seen that the use of three-dimensional technology in the anatomy of the duodenum and its surrounding organs still has a great impact on the operation. It has a certain degree of influence on the mainstream ideology of doctors. $85 \%$ of doctors believe that $3 \mathrm{D}$ technology has a great impact on the anatomy of the duodenum and its surrounding organs, and $12 \%$ of doctors believe that $3 \mathrm{D}$ technology is useful for the anatomy of the duodenum and its surrounding organs. $2 \%$ of doctors believe that $3 \mathrm{D}$ technology has little effect on the anatomy of the duodenum and its surrounding organs, and $1 \%$ of doctors believe that 3D technology has little effect on the duodenum and its surrounding organs. The anatomy performed has no effect. According to the results of this investigation, three-dimensional technology has a great impact on the anatomy of the duodenum and its surrounding organs. With the support of three-dimensional technology, doctors have reduced a lot during the operation. burden. Especially after the continuous development of 3D technology and some breakthrough progress, it gradually began to receive the attention of medical staff. Three-dimensional technology 
has brought great changes to the work of doctors. It is of great significance to use three-dimensional technology to better diagnose patients and perform preoperative analysis to improve the success efficiency of the operation.

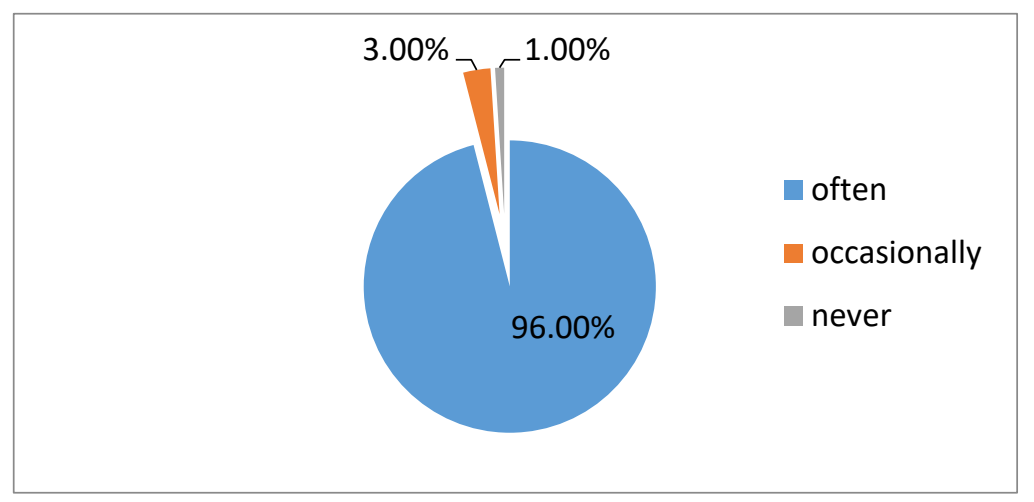

Figure 2. The use of 3D technology during surgery

From the data in Figure 2, it can be seen that the current anatomy of the duodenum and its surrounding organs is basically inseparable from three-dimensional technology. According to the data, $96 \%$ of the cases are in the duodenum and its surrounding organs. The three-dimensional technique is often used in the anatomy of the organ. In $3 \%$ of the cases, the three-dimensional technique is occasionally used in the anatomy of the duodenum and its surrounding organs, and in $1 \%$ of the cases. No need to use 3D technology. Three-dimensional technology is very important and positive for the anatomy of the duodenum and its surrounding organs. The use of this technology in the anatomy of the duodenum and its surrounding organs can greatly improve the success of the operation. rate.

\subsection{Advantages of 3D visualization technology in surgery}

(1) Three-dimensional visualization technology has unparalleled advantages in surgery. It can make accurate predictions before surgery. Using this technology can provide precise analysis of the duodenum and its surrounding organs and simulated surgery planning. Precise operation provides a solid foundation. During the operation, the doctor can remove the lesion to the maximum, avoid side injuries, and increase the success rate of the operation; (2) Perform accurate and differentiated data analysis on the duodenum and its surrounding organs, calculate the safe amount of resection, Before formulating an optimization ratio, different surgical plans are evaluated scientifically to evaluate the surgical risks. The safe cutting of the operation is also guaranteed. The doctor can formulate the best surgical plan, reduce the postoperative risk, and ensure the safety of the operation; (3) The construction of the three-dimensional structure allows the doctor to more intuitively analyze the adjacent relationship between the location of the lesion and the blood vessel before the operation, and grasp the exact position that needs to be removed, the size and number of the removal, improve the accuracy of the operation, and ensure the operation
Reasonableness. Make the preoperative surgical planning more precise; at the same time, the three-dimensional simulated surgical design is of great help to increase the confidence of young doctors and the surgical operation.

\section{Conclusions}

With the development of society, there are constant emergence of high technology. Under such a background, modern medicine is no longer limited to traditional backward medical technology. The emergence of three-dimensional technology is a major breakthrough in modern medical technology. This technology has many advantages in current medicine and can truly reflect the human body through virtual models. The anatomical structure and pathological characteristics. And three-dimensional technology also has huge applications in multi-directional observation. This function uses 3D printing technology to print out various virtual models, which has the advantages of being intuitive and not easily damaged. It can simulate the clinical work environment and allow students to have more exposure to the clinical environment in medical education, and smoothly transition to actual clinical work. It can be used as an important auxiliary means to be used in medical education to improve the level and quality of teaching. Three-dimensional technology has been proven to be beneficial to the development of medicine and education. At the same time, there is still an urgent need for more and deeper innovations and applications to promote the progress of my country's medical system and make medicine better serve the society and the public.

Foundation projects: The school-level first-class course project of Kunming Medical University in 2020 (Project number: 2020JXZ011)

Foundation projects: The school-level teaching research and reform project of Kunming Medical University ( Project number: 2020-JY-Y-008; 2020-JY-Y-011) foundation projects: The school-level quality project of Kunming Medical University in 2019 the construction of Human Anatomy blended curriculum 


\section{References}

1. Akiba Y, Kaunitz J D . Sodium Caprate Increases Electrogenic Anion Secretion via TRPV4, ATP cc 2Y and VIP $\neg$ PAC1 Pathways in Rat Duodenum[J]. The FASEB Journal, 2020, 34(S1):1-1.

2. Xiao Z L, Xu K S , Song Y H . Unusual cause of lesions in the descending duodenum and liver:A case report and review of literature[J]. World Journal of Clinical Cases, 2018, 6(11):71-75.

3. Kasendra M, Tovaglieri A, Sontheimer-Phelps A, et al. Development of a primary human Small Intestine-on-a-Chip using biopsy-derived organoids[J]. Rep, 2018, 8(1):2871.

4. Trukhmanov A S, Ivashkina N Y I . The clinical significance of disorders of the motor function of the esophagus, stomach and duodenum[J]. Terapevticheskii arkhiv, 2019, 91(8):127-134.

5. Hendriks S, Verseveld M M , Egbert Roeland Boevé, et al. Successful endoscopic treatment of a large impacted gallstone in the duodenum using laser lithotripsy, Bouveret's syndrome: A case report[J]. World Journal of Gastroenterology, 2020, 26(19):2457-2462.

6. Bein A, Shin W, Jalili-Firoozinezhad S, et al. Microfluidic Organ-on-a-Chip Models of Human Intestine[J]. Cellular and Molecular Gastroenterology and Hepatology, 2018, 5(4):659-668.

7. Mingming W, Yajie W, Ranran P, et al. Genome-Wide Identification and Characterization of MADS-box Family Genes Related to Floral Organ Development and Stress Resistance in Hevea brasiliensis Müll. Arg[J]. Forests, 2018, 9(6):304-.

8. Chen, Zhonghua $\mathrm{K}$, Zeng, et al. CURRENT SITUATION OF ORGAN DONATION IN CHINA FROM STIGMA TO STIGMATA.[J]. Doklady Earth ences, 2018, 443(2):439-443.

9. Uryuhara Y . Success Factors for Social Systems to Increase the Number of Organ Donations- From the Perspectives of Mechanisms and Organizational Behaviors[J]. International Journal of Clinical Medicine, 2018, 9(2):59-70.

10. Rainieri S, Conlledo N, Larsen B K, et al. Combined effects of microplastics and chemical contaminants on the organ toxicity of zebrafish ( Danio rerio ) [J]. Environmental Research, 2018, 162(APR.):135-143. 